\title{
A quantitative and in-depth survey of the isotopic abundance distribution errors in shotgun proteomics
}

Cheng Chang ${ }^{1 \#}$, Jiyang Zhang ${ }^{2 \#}$, Changming $\mathrm{Xu}^{2}$, Yan Zhao ${ }^{1}, \mathrm{Jie} \mathrm{Ma}^{1}$, Tao Chen ${ }^{1}$, Fuchu $\mathrm{He}^{1^{*}}$, Hongwei Xie ${ }^{2^{*}}$, Yunping $\mathrm{Zhu}^{1^{*}}$

${ }^{1}$ State Key Laboratory of Proteomics, Beijing Proteome Research Center, National Engineering Research Center for Protein Drugs, National Center for Protein Sciences (Beijing), Beijing Institute of Radiation Medicine, Beijing, 102206, P.R.

China.

${ }^{2}$ Department of Automatic Control, College of Mechanical Engineering and Automation, National University of Defense Technology, Changsha, Human, 410073, P.R. China.

\# The first two authors contributed equally to this work and share first authorship.

\section{Corresponding Authors}

*(Y.Z.) E-mail: zhuyunping@gmail.com; Tel and Fax: +86-10-61777058.

*(H.X.) Email: $\quad$ xhwei65@163.com; Tel: +86-731-84576311; Fax: $+86-731-84576311$.

*(F.H.) E-mail: hefc@ nic.bmi.ac.cn; Tel and Fax: +86-10-68171208. 


\section{Supporting Information and Methods}

\section{Cell Lysis and Protein Digestion}

Yeast (S. cerevisiaestrain) strains BY4743 and macrophage cells from Mus musculus RAW264.7 were lysed in lysis buffer $(8 \mathrm{M}$ urea, $100 \mathrm{mM}$ Tris, $\mathrm{pH} 8.0$ solution containing $1 \%$ protease inhibitor cocktail) and disrupted by sonication (Ningbo Scientz Biotechnology CO.LTD, China). The lysate were prepared by centrifugation at $14,000 \mathrm{~g}, 4{ }^{\circ} \mathrm{C}$. Reduction and alkylation of proteins were performed successively by the addition of dithiothreitol at the final concentration of $10 \mathrm{mM}$ and iodine acetamide at the final concentration of $25 \mathrm{mM}$. The lysate was diluted 1:10 with $50 \mathrm{mM}$ ammonium bicarbonate for tryspsin-catalysed digestion. After incubation overnight at $37^{\circ} \mathrm{C}$, the digest was acidified with trifluoroacetic acid and desalted for further analysis.

\section{LC-MS/MS Analysis}

The peptides were loaded and separated on a $15 \mathrm{~cm} 75 \mu \mathrm{m}$ i.d column packed in-house with $\mathrm{C}_{18}$ resin $\left(\mathrm{C}_{18}\right.$ AQ $3 \mu \mathrm{m}$, Agela, China). The chromatographic gradient was controlled by Easy-nLC 1000 (Proxeon, Odense, Denmark) system as following: phase $\mathrm{B}(100 \% \mathrm{ACN}$ and $0.1 \%$ formic acid) increased from $5 \%$ to $8 \%$ in $8 \mathrm{~min}, 8 \%$ to $22 \%$ in $50 \mathrm{~min}$ and ramped to $32 \% \mathrm{~B}$ in $12 \mathrm{~min}$ at $300 \mathrm{nl} / \mathrm{min}$. MS data were acquired by the Q-Exactive Orbitrap mass spectrometer (Thermo Fisher Scientific) operated in a data-dependent mode. The parameters of MS full scan: resolution, 70,000 at $\mathrm{m} / \mathrm{z}=200$; AGC value, $3 \times 10^{6}$; maximum fill time, $60 \mathrm{~ms}$. The ions in the range of $300-1400 \mathrm{~m} / \mathrm{z}$ were isolated and fragmented by HCD at normalized collision energy 
of $27 \mathrm{eV}$. Top $20 \mathrm{MS} / \mathrm{MS}$ spectra were acquired: resolution, 17,500 at m/z=200; AGC value, $5 \times 10^{4}$; maximum fill time, $80 \mathrm{~ms} .3 \mathrm{~m} / \mathrm{z}$ window was set to isolate precursors. The time for dynamically exclusion of MS/MS spectra acquisition was $18 \mathrm{~s}$.

\section{Two Additional Isotopic Abundance Models}

Since the theory of FTMS-based instruments include analog signal sampling and amplification, we defined two additional models to be used in many physical measurement processes for isotopic profile abundance errors.

The first model (M1) is called the "additive error model". As shown in equations 1 and 2, we assumed that the observed isotopic profile is the amplification of the theoretical isotopic distribution with additive random errors. All of the peaks were amplified with the same gain.

$$
\begin{gathered}
I_{E}^{i}=A I_{T}^{i}+\varepsilon_{i} \\
\text { Error }_{i}=\varepsilon_{i} / I_{E}^{i}
\end{gathered}
$$

where $I_{E}^{i}$ and $I_{T}^{i}$ denote the experimental and theoretic abundance of the $i^{\text {th }}$ isotopic peak for a given isotopic cluster, $\varepsilon_{i}$ is a small random error, and $A$ is amplification gain.

The second model (M2) is called the "multiplicative error model" which assumes that the peaks in the isotopic profile are amplified with gains including random errors (equations 3 and 4).

$$
\begin{aligned}
& I_{E}^{i}=\left(A+\varepsilon_{i}\right) I_{T}^{i} \\
& \text { Error }_{i}=\varepsilon_{i} / A
\end{aligned}
$$

where $I_{E}^{i}$ and $I_{T}^{i}$ denote the experimental and theoretic abundance of the $i^{\text {th }}$ 
isotopic peak for a given isotopic cluster, $\varepsilon_{i}$ is a small random error, and $A$ is amplification gain. 


\section{Supplementary Figures and Tables}

\section{List of Figures}

Figure S1. Scatterplots of the isotopic peak abundance and its error for the four concentration levels A-D (a-d) in dataset D1.

Figure S2. Scatterplots of mono-isotope mass (Da) and intensity (logarithmic transformed) in two groups for all the isotopic clusters with different isotope numbers (IsoNum3-6) in dataset D2.

Figure S3. Scatterplots of mono-isotope mass (Da) and intensity (logarithmic transformed) in two groups for all the isotopic clusters with different isotope numbers (IsoNum3-6) in dataset D3.

Figure S4. Boxplots of the mono-isotope mass (Da) for isotopic clusters with different peak numbers (3-6) for datasets D1-D3 (a-c).

Figure S5. Boxplots of the mono-isotope retention time (minute) for isotopic clusters with different peak numbers (3-6) for datasets D1-D3 (a-c).

Figure S6. Boxplots of the mono-isotope intensity (logarithmic transformed) for isotopic clusters with different peak numbers (3-6) for datasets D1-D3 (a-c). 
Figure S7. Boxplots of the mono-isotope S/N (logarithmic transformed) for isotopic clusters with different peak numbers (3-6) for datasets D1-D3 (a-c).

Figure S8. Boxplots of the mono-isotope mass error ( $\mathrm{ppm}$ ) for isotopic clusters with different peak numbers (3-6) for datasets D1-D3 (a-c).

Figure S9. Boxplots of the mono-isotope abundance error for isotopic clusters with different peak numbers (3-6) for datasets D1-D3 (a-c).

Figure S10. Heatmap of Pearson correlation coefficients for the mono-isotope errors of all the isotopic clusters with different peak numbers (IsoNum 3-6) in dataset D1.

Figure S11. Heatmap of Pearson correlation coefficients for the mono-isotope errors of all the isotopic clusters with different peak numbers (IsoNum 3-6) in dataset D2.

Figure S12. Heatmap of Pearson correlation coefficients for the mono-isotope errors of all the isotopic clusters with different peak numbers (IsoNum 3-6) in dataset D3. 
Figure S1. Scatterplots of the isotopic peak abundance and its error for the four concentration levels A-D (a-d) in dataset D1.

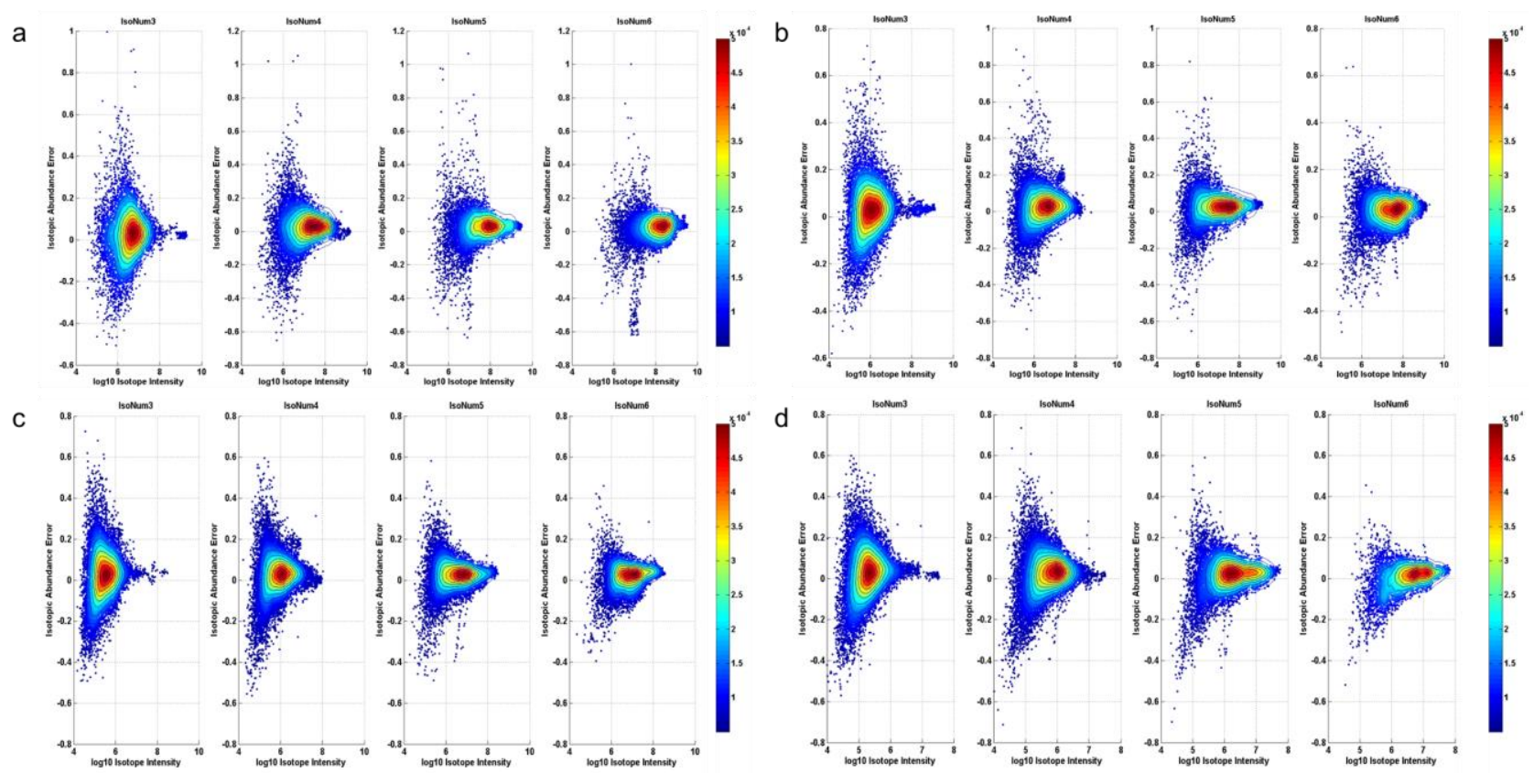


Figure S2. Scatterplots of mono-isotope mass (Da) and intensity (logarithmic transformed) in two groups for all the isotopic clusters with different isotope numbers (IsoNum3-6) in dataset D2. A-D indicate the four levels of dilution concentrations in each dataset. The blue hollow circles represent the mono-isotope which is the most intense peak in one isotopic cluster. The red ones represent the mono-isotope which is not the most intense peak in one isotopic cluster. The black dashed line means the linear regression line in either group.

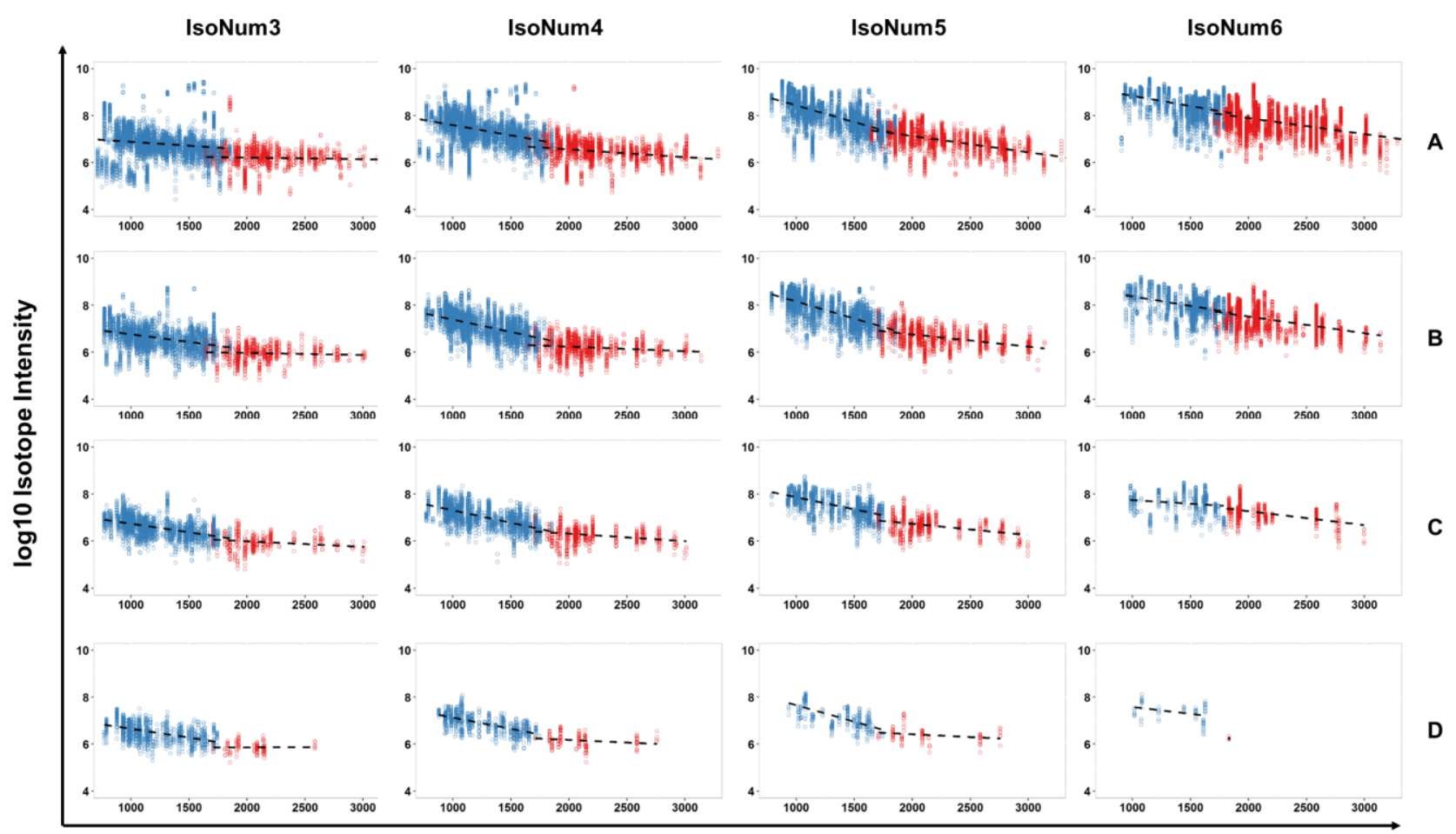

Mass (Da) 
Figure S3. Scatterplots of mono-isotope mass (Da) and intensity (logarithmic transformed) in two groups for all the isotopic clusters with different isotope numbers (IsoNum3-6) in dataset D3. A-D indicate the four levels of dilution concentrations in each dataset. The blue hollow circles represent the mono-isotope which is the most intense peak in one isotopic cluster. The red ones represent the mono-isotope which is not the most intense peak in one isotopic cluster. The black dashed line means the linear regression line in either group.

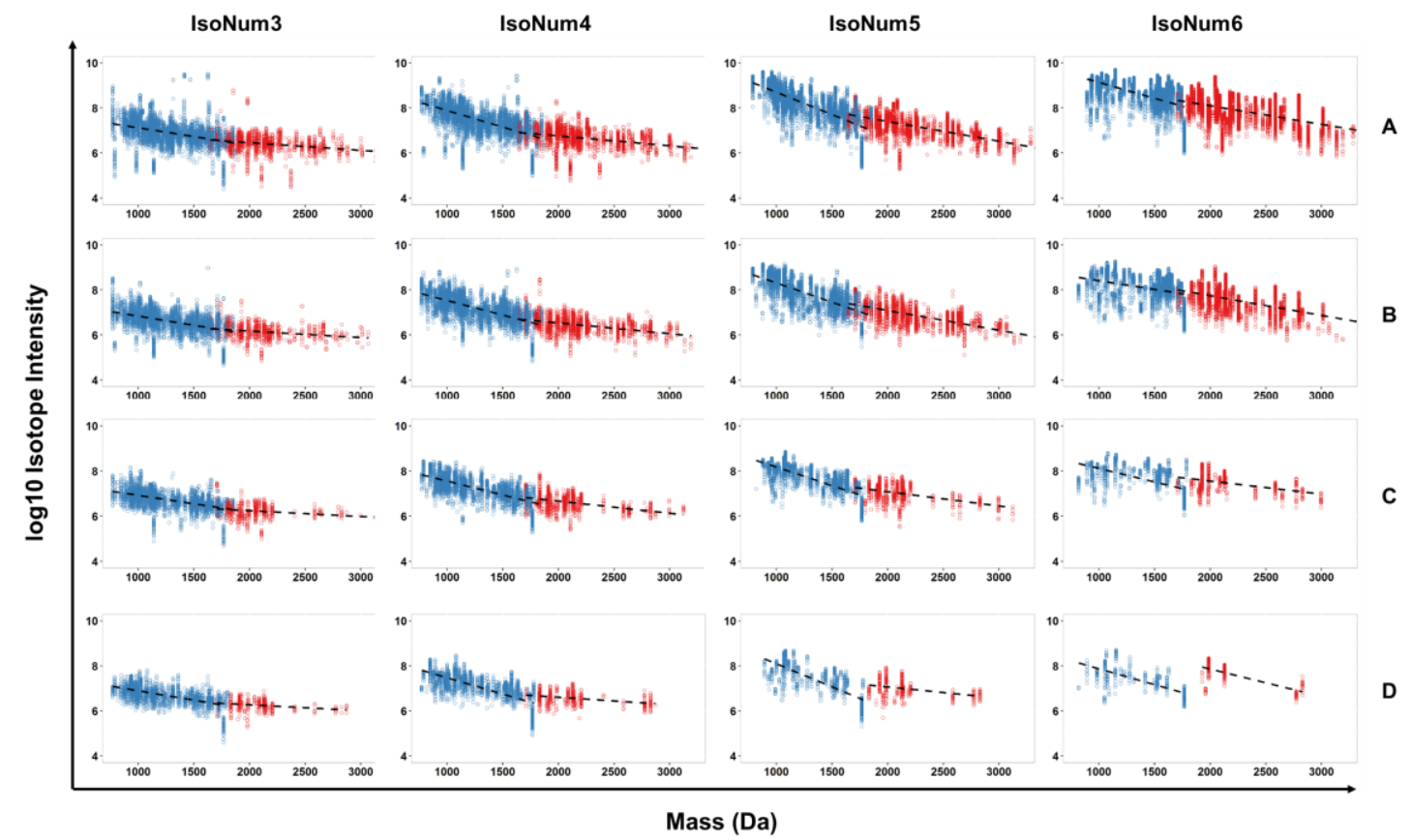


Figure S4. Boxplots of the mono-isotope mass (Da) for isotopic clusters with different peak numbers (3-6) for datasets D1-D3 (a-c). A-D indicate the four levels of dilution concentrations in each dataset.

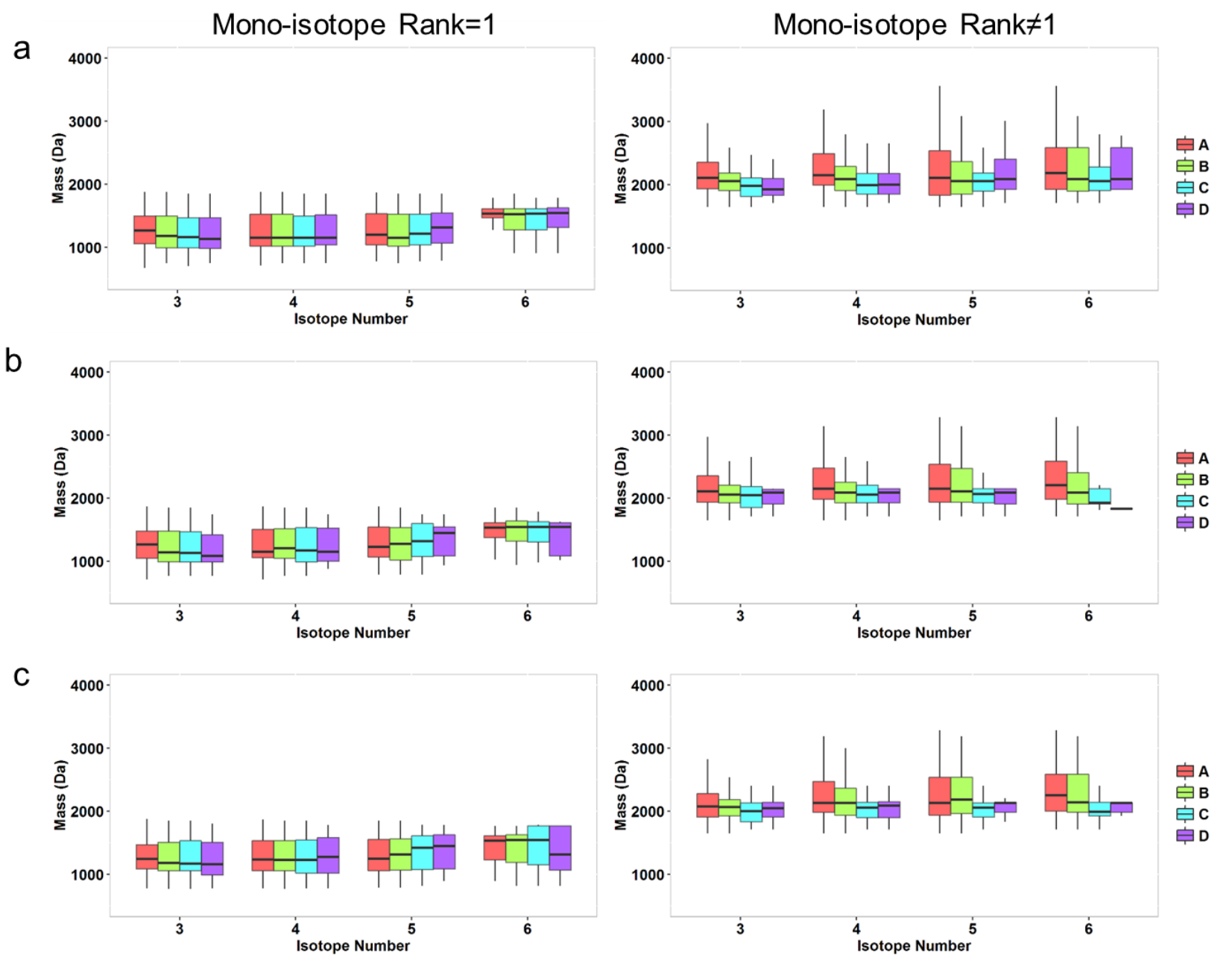


Figure S5. Boxplots of the mono-isotope retention time (minute) for isotopic clusters with different peak numbers (3-6) for datasets D1-D3 (a-c). A-D indicate the four levels of dilution concentrations in each dataset.

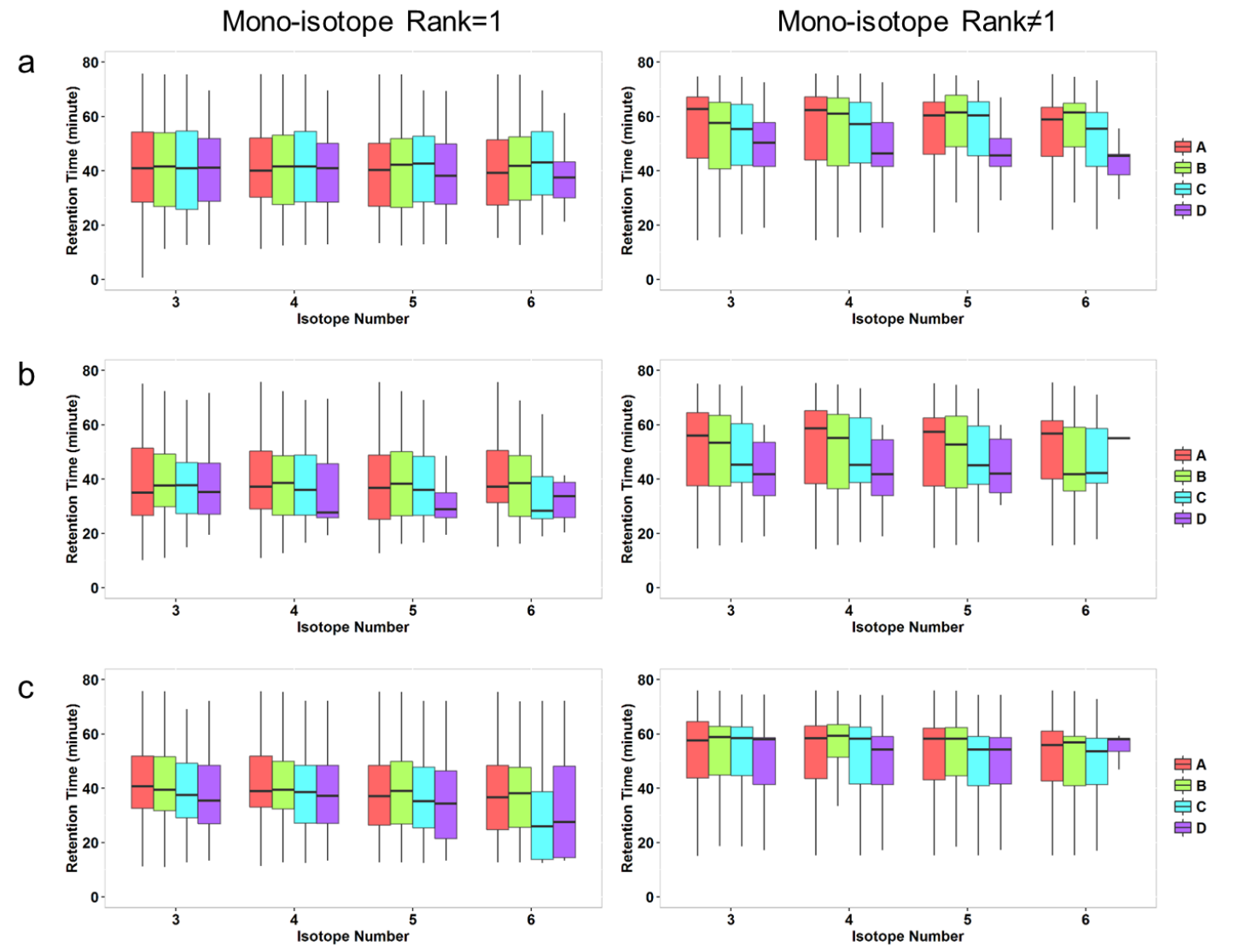


Figure S6. Boxplots of the mono-isotope intensity (logarithmic transformed) for isotopic clusters with different peak numbers (3-6) for datasets D1-D3 (a-c). A-D indicate the four levels of dilution concentrations in each dataset.

a
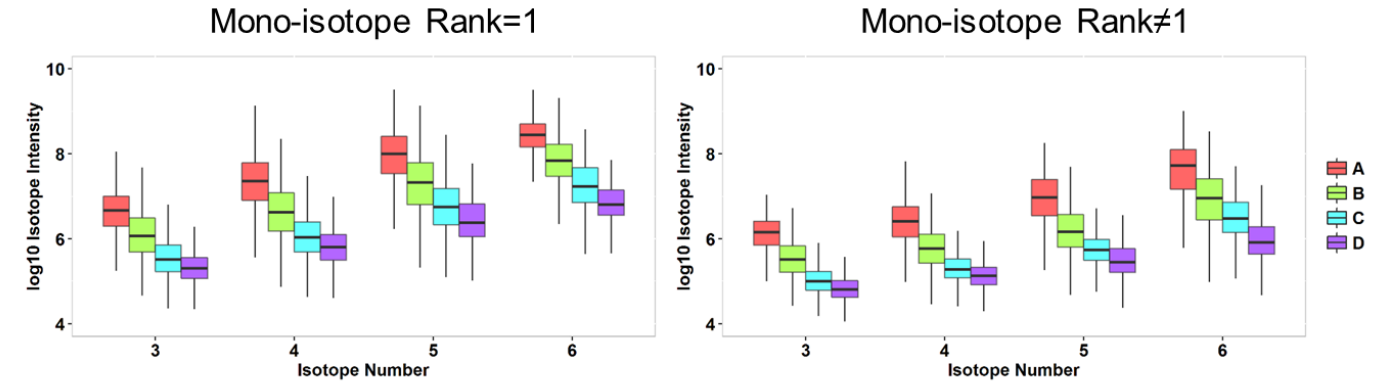

b
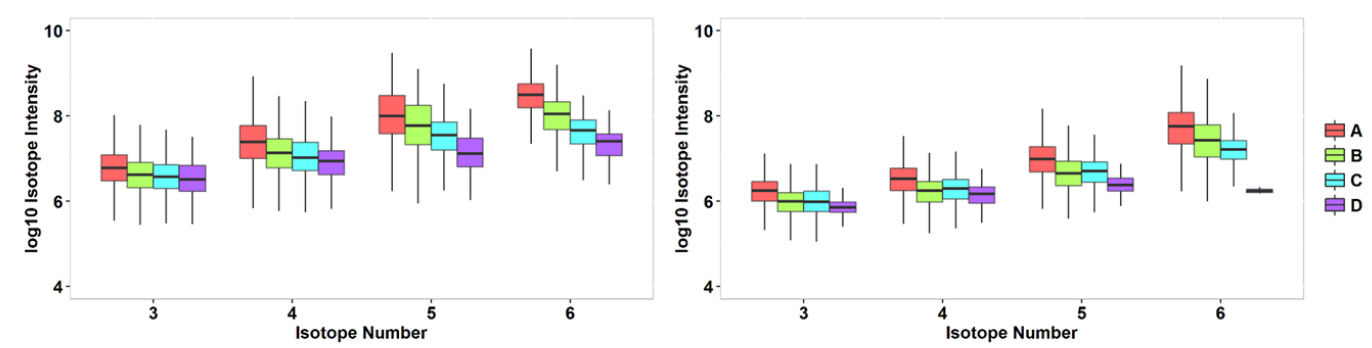

C
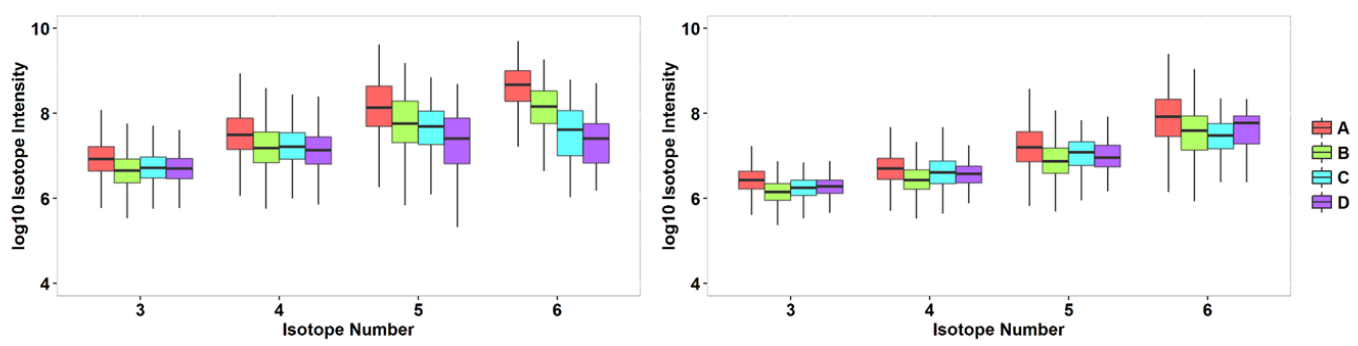
Figure S7. Boxplots of the mono-isotope S/N (logarithmic transformed) for isotopic clusters with different peak numbers (3-6) for datasets D1-D3 (a-c). A-D indicate the four levels of dilution concentrations in each dataset.
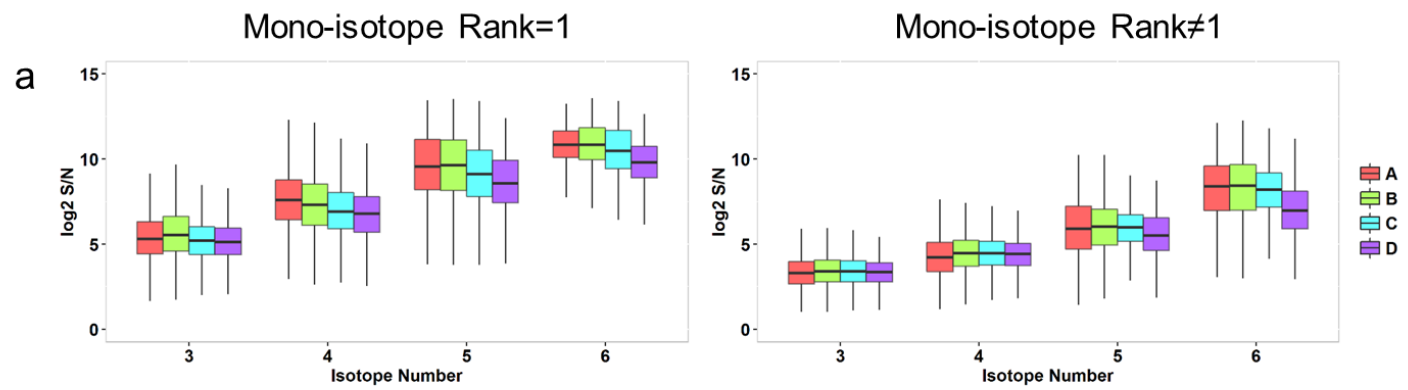

b
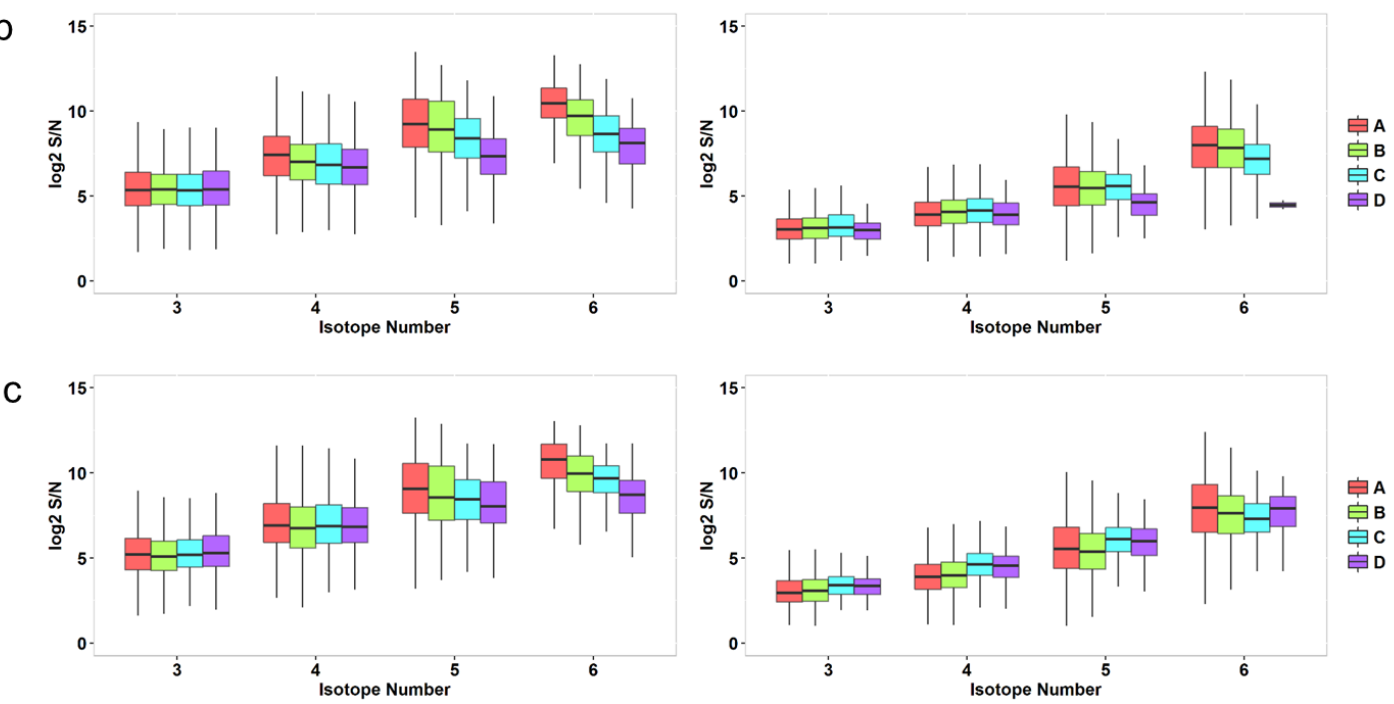
Figure S8. Boxplots of the mono-isotope mass error (ppm) for isotopic clusters with different peak numbers (3-6) for datasets D1-D3 (a-c). A-D indicate the four levels of dilution concentrations in each dataset.
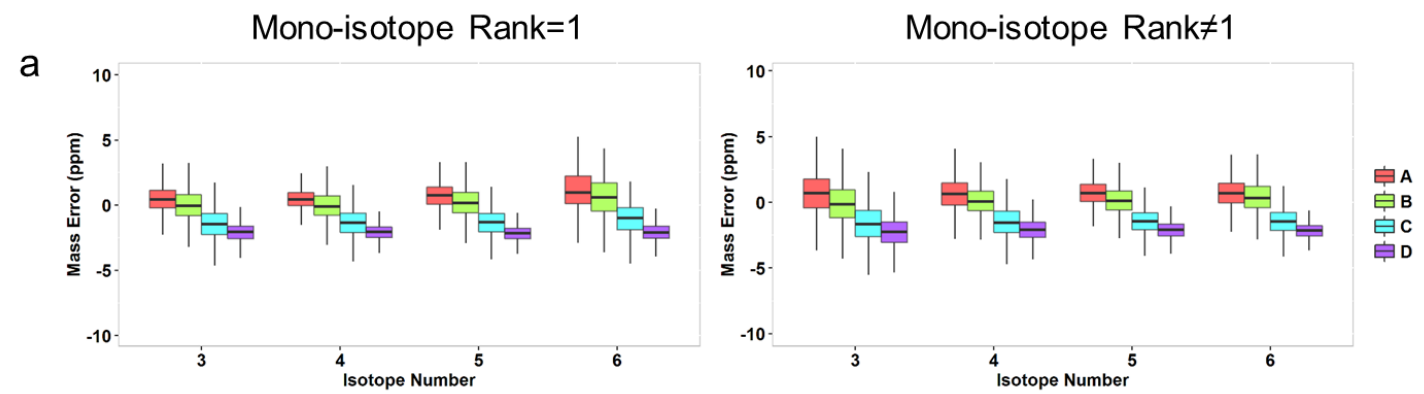

b
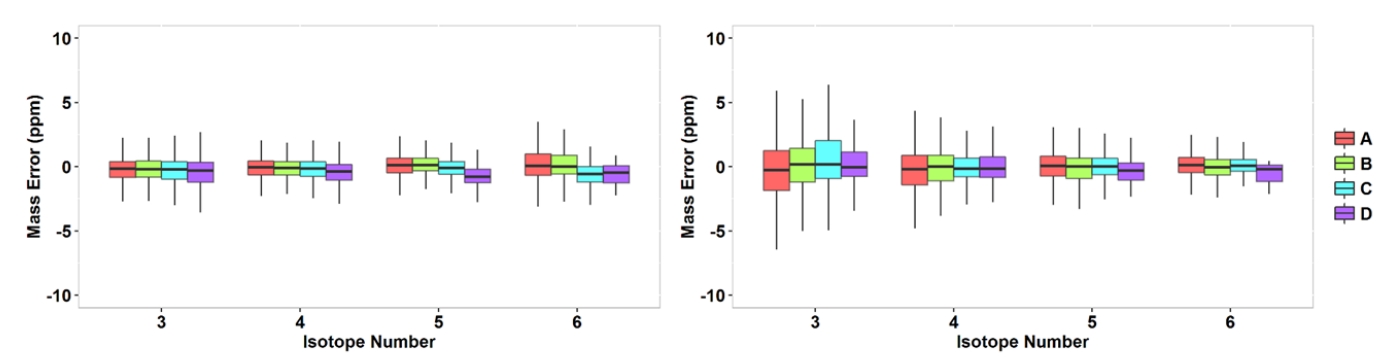

C
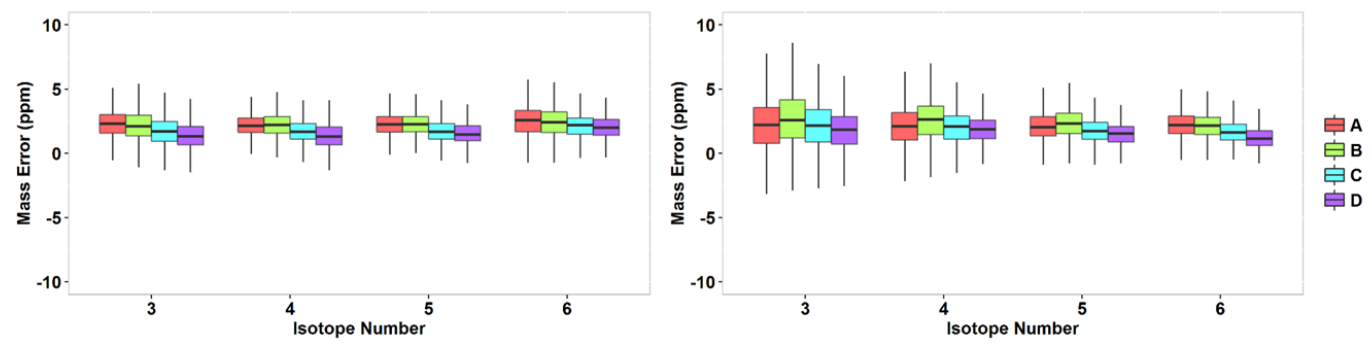
Figure S9. Boxplots of the mono-isotope abundance error for isotopic clusters with different peak numbers (3-6) for datasets D1-D3 (a-c). A-D indicate the four levels of dilution concentrations in each dataset.
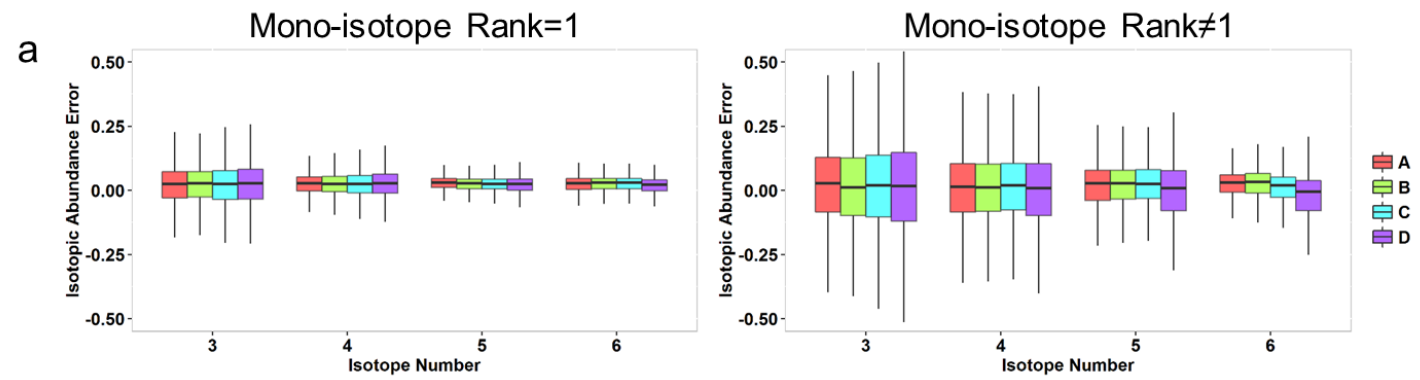

b
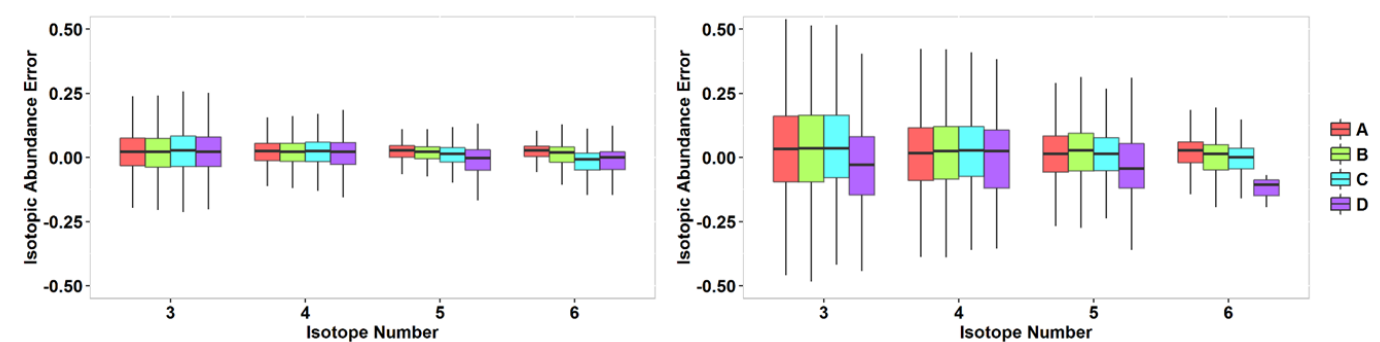

C
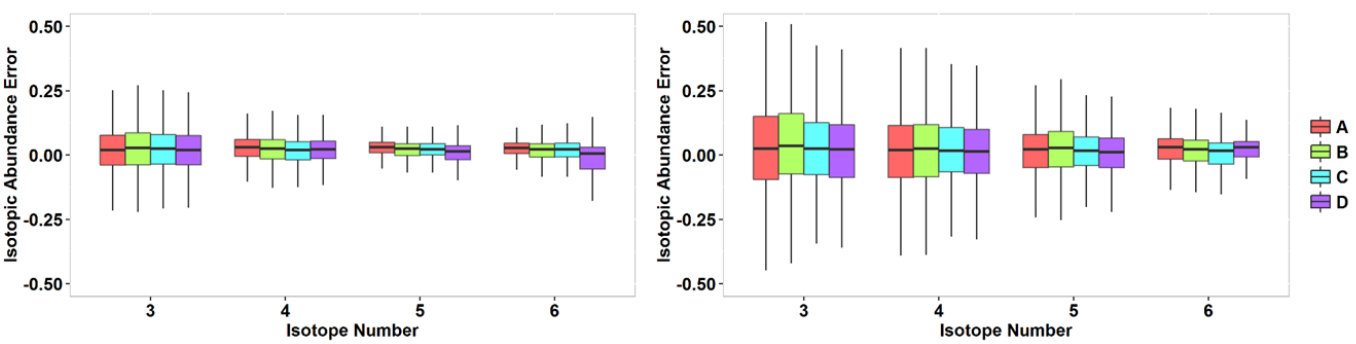
Figure S10. Heatmap of Pearson correlation coefficients for the mono-isotope errors of all the isotopic clusters with different peak numbers (IsoNum 3-6) in dataset D1.

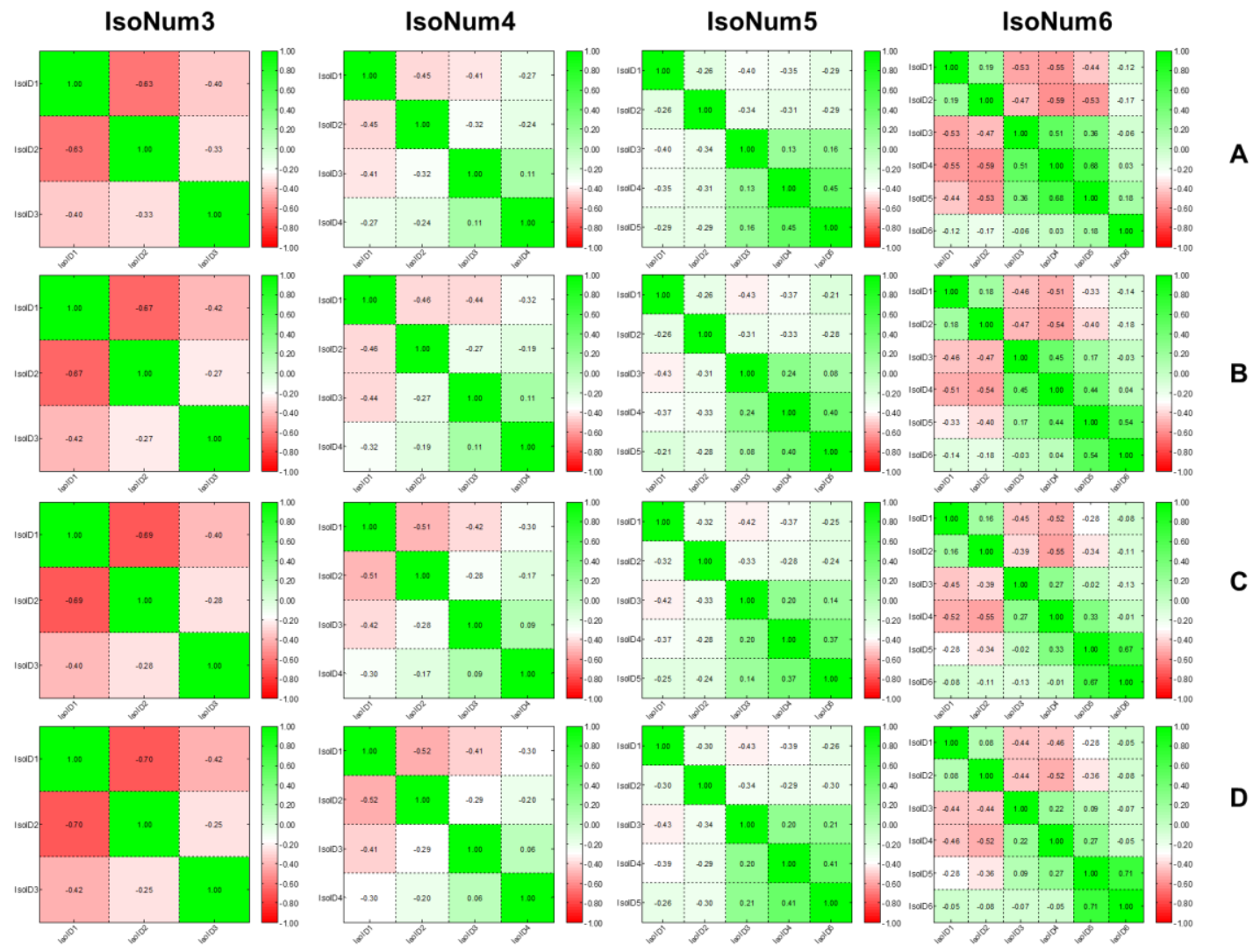


Figure S11. Heatmap of Pearson correlation coefficients for the mono-isotope errors of all the isotopic clusters with different peak numbers (IsoNum 3-6) in dataset D2.

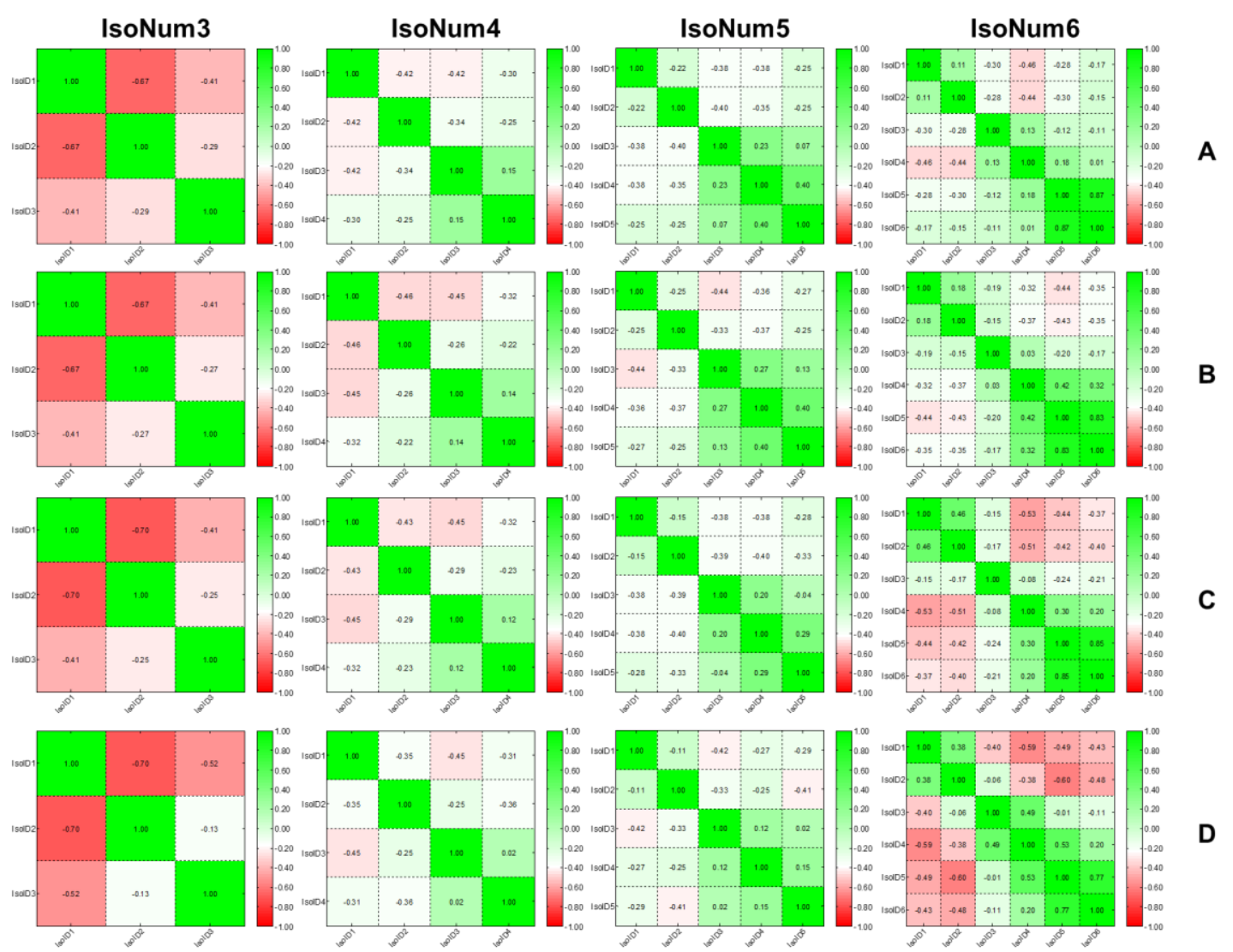


Figure S12. Heatmap of Pearson correlation coefficients for the mono-isotope errors of all the isotopic clusters with different peak numbers (IsoNum 3-6) in dataset D3.

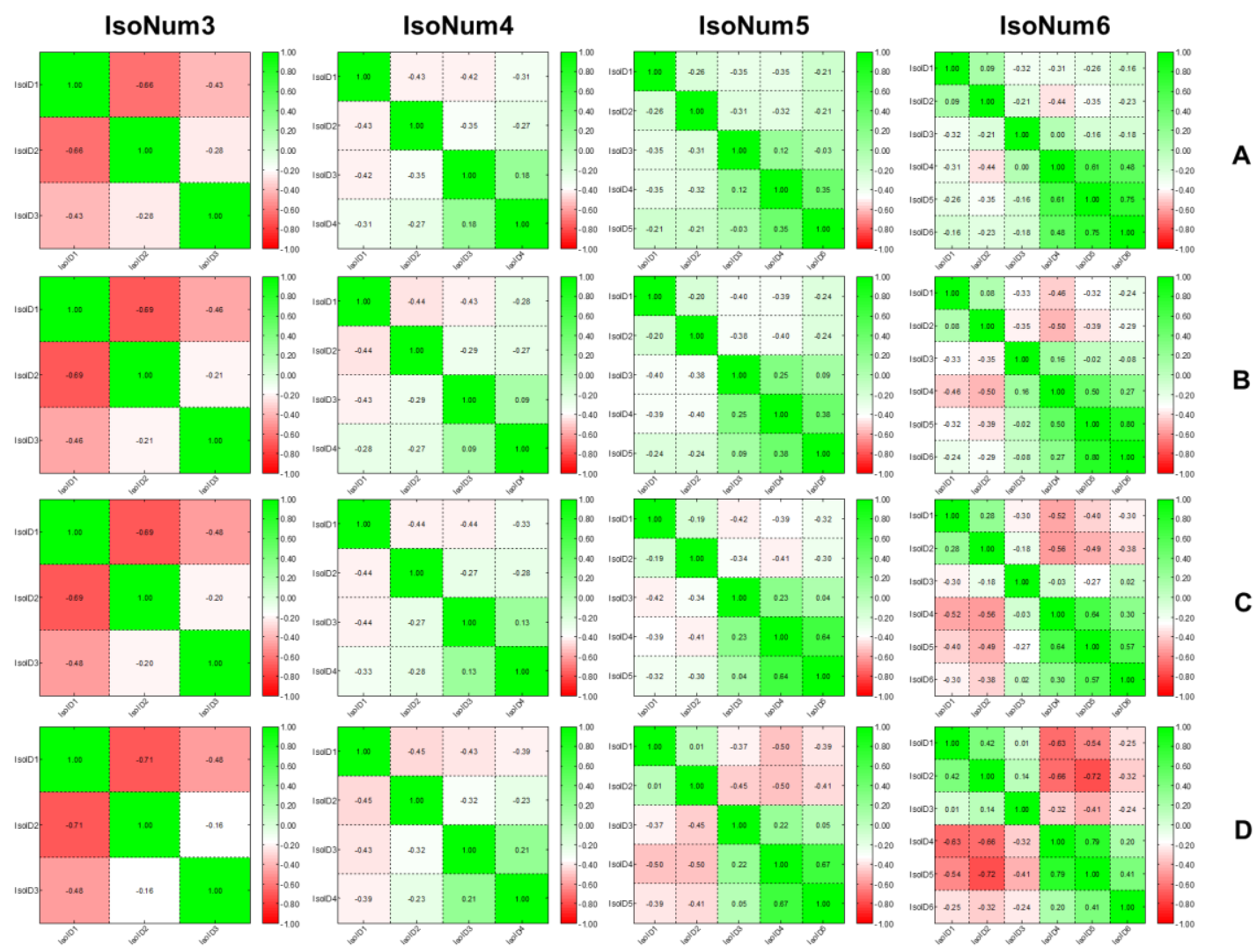




\section{List of Tables}

Table S1. The detailed information and search parameters of the three UPS2 spiked-in datasets (D1-D3) in this study.

Table S2. The isotope peak number summary in the three UPS2 spiked-in datasets (D1-D3). A-D indicate the four levels of dilution concentrations in each dataset.

Table S3. Statistics of the RIA deviation for isotopic peak A+1 to A+5 (the IsoID2-6 peaks versus the mono-isotopic peak) for three UPS2 spiked-in datasets (D1-D3). A-D indicate the four levels of dilution concentrations in each dataset. 
Table S1. The detailed information and search parameters of the three UPS2 spiked-in datasets and six public datasets (D1-D3) in this study.

\begin{tabular}{ccccccc}
\hline Dataset & Instrument & File number & Fragmentation & Enzyme & $\begin{array}{c}\text { Precursor ion } \\
\text { tolerance(ppm) }\end{array}$ & $\begin{array}{c}\text { Product ion } \\
\text { tolerance(Da) }\end{array}$ \\
\hline D1 & Q-Exactive & 12 & HCD & Trypsin & 20 & 0.05 \\
D2 & Q-Exactive & 12 & HCD & Trypsin & 20 & 0.05 \\
D3 & Q-Exactive & 12 & HCD & Trypsin & 20 & 0.05 \\
\hline
\end{tabular}

Note: the corresponding fix and dynamic modifications in D1 D9 were Carbamidomethylation cysteine (+57.0215Da) and oxidation (+15.9949Da) on methionine, respectively.

Table S2. The isotope peak number summary in the three UPS2 spiked-in datasets (D1-D3). A-D indicate the four levels of dilution concentrations in each dataset.

\begin{tabular}{|c|c|c|c|c|c|c|c|c|c|c|c|c|}
\hline \multirow{2}{*}{ IsoID } & \multicolumn{4}{|c|}{ D1 } & \multicolumn{4}{|c|}{ D2 } & \multicolumn{4}{|c|}{ D3 } \\
\hline & A & B & $\mathrm{C}$ & $\mathrm{D}$ & A & B & $\mathrm{C}$ & $\mathrm{D}$ & A & B & $\mathrm{C}$ & $\mathrm{D}$ \\
\hline 1 & 45142 & 52920 & 57344 & 50701 & 36831 & 22593 & 11360 & 3288 & 41769 & 25617 & 13851 & 7703 \\
\hline 2 & 45142 & 52920 & 57344 & 50701 & 36831 & 22593 & 11360 & 3288 & 41769 & 25617 & 13851 & 7703 \\
\hline 3 & 45142 & 52920 & 57344 & 50701 & 36831 & 22593 & 11360 & 3288 & 41769 & 25617 & 13851 & 7703 \\
\hline 4 & 37021 & 41192 & 44417 & 36255 & 29006 & 17501 & 7920 & 1735 & 34527 & 20609 & 9951 & 5041 \\
\hline 5 & 24697 & 26189 & 26933 & 17258 & 19592 & 11185 & 4298 & 550 & 24965 & 13640 & 5541 & 2127 \\
\hline 6 & 12978 & 12521 & 11354 & 5027 & 11172 & 5533 & 1390 & 84 & 14750 & 7062 & 2096 & 735 \\
\hline
\end{tabular}

Table S3. Statistics of the RIA deviation for isotopic peak A+1 to A+5 (the IsoID2-6

peaks versus the mono-isotopic peak) for three UPS2 spiked-in datasets (D1-D3).

A-D indicate the four levels of dilution concentrations in each dataset.

\begin{tabular}{lcrrrrrrrrrr}
\hline \multirow{2}{*}{ Dataset } & & \multicolumn{2}{c}{ A+1 } & \multicolumn{2}{c}{ A+2 } & \multicolumn{2}{c}{ A+3 } & \multicolumn{2}{c}{ A+4 } & \multicolumn{2}{c}{ A+5 } \\
\cline { 2 - 13 } & & median & SD & median & SD & median & SD & median & SD & median & SD \\
\hline \multirow{4}{*}{ D1 } & A & 5.65 & 14.93 & 3.78 & 13.04 & 1.91 & 8.98 & 0.87 & 5.56 & 0.42 & 3.07 \\
& B & 5.66 & 12.93 & 3.60 & 10.52 & 1.78 & 7.57 & 0.81 & 4.76 & 0.43 & 3.51 \\
& C & 5.87 & 12.85 & 3.62 & 9.40 & 1.69 & 6.18 & 0.73 & 3.66 & 0.41 & 2.92 \\
& D & 6.66 & 14.28 & 3.83 & 10.79 & 1.75 & 7.92 & 0.80 & 5.18 & 0.55 & 3.56 \\
D2 & A & 6.17 & 14.86 & 4.08 & 12.34 & 2.10 & 9.38 & 1.05 & 7.57 & 0.55 & 5.76 \\
& B & 6.08 & 15.24 & 4.01 & 11.21 & 2.12 & 7.82 & 1.06 & 5.58 & 0.68 & 5.53 \\
& C & 6.90 & 13.20 & 4.11 & 10.59 & 2.06 & 7.89 & 1.19 & 6.71 & 1.10 & 7.42 \\
& D & 7.44 & 14.71 & 4.43 & 11.63 & 2.03 & 8.55 & 2.50 & 9.79 & 1.38 & 9.41
\end{tabular}




\begin{tabular}{rrrrrrrrrrrr} 
& A & 6.15 & 14.88 & 4.16 & 12.68 & 2.17 & 9.88 & 0.97 & 7.90 & 0.47 & 6.95 \\
D3 & B & 6.39 & 17.53 & 4.32 & 12.37 & 2.31 & 9.76 & 1.15 & 5.92 & 0.62 & 5.57 \\
& C & 6.14 & 12.22 & 3.93 & 9.08 & 1.88 & 6.94 & 0.98 & 4.74 & 0.58 & 3.74 \\
& D & 6.56 & 12.66 & 4.02 & 9.44 & 2.04 & 7.69 & 1.31 & 5.74 & 0.90 & 5.62 \\
\hline
\end{tabular}

